\title{
APPROXIMATION BY NÖRLUND AND RIESZ TYPE DEFERRED CESÀRO MEANS IN THE SPACE $H_{P}^{(\omega)}$
}

\author{
UǦUR DEǦER AND HILAL BAYINDIR
}

Received 15 February, 2017

\begin{abstract}
The deferred Cesàro transformations which have useful properties not possessed by the Cesàro transformation was considered by R.P. Agnew in [1]. In [9], Değer and Küçükaslan introduced a generalization of deferred Cesàro transformations by taking account of some well known transformations such as Woronoi-Nörlund and Riesz, and considered the degree of approximation by the generalized deferred Cesàro means in the space $H(\alpha, p), p \geq 1,0<\alpha \leq 1$ by concerning with some sequence classes. In 2014, Nayak et al. studied the rate of convergence problem of Fourier series by deferred Cesàro mean in the space $H_{p}^{(\omega)}$ introduced by Das et al. in [7].

In this study we shall give the degree of approximation by the generalized deferred Cesàro means in the space $H_{p}^{(\omega)}$. Therefore the results given in [17] are generalized according to the summability method.
\end{abstract}

2010 Mathematics Subject Classification: 40G05; 41A25; 42A05; 42A10

Keywords: deferred Cesàro means, Nörlund means, generalized Hölder metric

\section{INTRODUCTION}

Let $f$ be a $2 \pi$ - periodic function and $f \in L_{p}:=L_{p}(0,2 \pi)$ for $p \geq 1$ and let

$$
s_{n}(f ; x)=\frac{1}{2} a_{0}+\sum_{k=1}^{n}\left(a_{k} \cos k x+b_{k} \sin k x\right) \equiv \sum_{k=0}^{n} A_{k}(f ; x)
$$

partial sum of the first $(n+1)$ terms of the Fourier series of $f \in L_{p}(p \geq 1)$ at a point $x$.

The degree of approximation of the sums $s_{n}(f ; x)$ in different spaces has been studied by many authors. In [20], Quade investigated approximation properties of the partial sums of Fourier series in $L_{p}$ norms. Chandra in [5] and Leindler in [13] generalized the results of Quade using the Woronoi-Nörlund and the Riesz means of Fourier series for some sequence classes. A similar problems were studied for more general means in [8] and [15]. 
The space

$$
H_{\alpha}=\left\{f \in C_{2 \pi}:|f(x)-f(y)| \leq K|x-y|^{\alpha}, 0<\alpha \leq 1\right\}
$$

where $C_{2 \pi}$ is space of all $2 \pi$-periodic and continuous functions defined on $\mathbb{R}$ with the supremum norm and $K$ is a positive constant, not necessarily the same at each occurrence, is a Banach space (see Prösdorff, [19]) with the norm $\|\cdot\|_{\alpha}$ defined by

$$
\|f\|_{\alpha}=\|f\|_{C}+\sup _{x \neq y} \Delta^{\alpha} f(x, y)
$$

where

$$
\Delta^{\alpha} f(x, y)=\frac{|f(x)-f(y)|}{|x-y|^{\alpha}}(x \neq y),
$$

by convention $\Delta^{0} f(x, y)=0$ and

$$
\|f\|_{C}=\sup _{x \in[-\pi, \pi]}|f(x)| .
$$

The metric generated by the norm (1.1) on $H_{\alpha}$ is called the Hölder metric. Prösdorff has studied the degree of approximation by Cesàro means in the Hölder metric and proved the following theorem.

Theorem 1 ([19]). Let $f \in H_{\alpha}(0<\alpha \leq 1)$ and $0 \leq \beta<\alpha \leq 1$. Then

$$
\left\|\sigma_{n}(f)-f\right\|_{\beta}=O(1) \begin{cases}n^{\beta-\alpha} & , 0<\alpha<1 ; \\ n^{\beta-1} \ln n & , \alpha=1\end{cases}
$$

where $\sigma_{n}(f)$ is the Cesàro means of the Fourier series of $f$.

The case $\beta=0$ in Theorem 1 is due to Alexits [2]. Leindler introduced more general classes than the Hölder classes of $2 \pi$-periodic continuous functions, generalized the results of Prösdorff [11]. Chandra obtained a generalization of Theorem 1 by considering the Woronoi-Nörlund transform [4]. In [16] Mohapatra and Chandra considered the problem by a matrix means of the Fourier series of $f \in H_{\alpha}$.

Further generalizations of the Hölder metric was given in [6] and [7]. In [6], Das et al. studied the results regarding the degree of approximation by infinite matrix means involved in the deferred Cesàro means in a generalized Hölder metric.

In [9], the authors considered the degree of approximation to functions in this space with respect to the norm in the space given in [6] by the deferred WoronoiNörlund means and the deferred Riesz means of the Fourier series of the functions.

The modulus of continuity of $f \in C_{2 \pi}$ is defined by

$$
\omega(f, \delta):=\sup _{0<|h| \leq \delta}|f(x+h)-f(x)| .
$$

According to this, the class of functions $H^{\omega}$ is defined by

$$
H^{\omega}:=\left\{f \in C_{2 \pi}: \omega(f, \delta)=O(\omega(\delta))\right\}
$$

where $\omega(\delta)$ is a modulus of continuity. 
There are numerous papers related to the degree of approximation in $H^{\omega}$ space such as Leindler [11], Mazhar and Totik [14], Das et al. [7], Khatri and Mishra [10].

A further generalization of $H^{\omega}$ space has been given by Das, Nath and Ray in [7]. They defined the following notations: If $f \in L_{p}(0,2 \pi), p \geq 1$, then denote

$$
H_{p}^{(\omega)}:=\left\{f \in L_{p}(0,2 \pi), p \geq 1: A(f, \omega)<\infty\right\}
$$

where $\omega$ is a modulus of continuity and

$$
A(f, \omega):=\sup _{t \neq 0} \frac{\|f(\cdot+t)-f(\cdot)\|_{p}}{\omega(|t|)}
$$

where $\|\cdot\|_{p}$ will denote $L_{p}$-norm with respect to $x$ and is defined by

$$
\|f\|_{p}:=\left\{\frac{1}{2 \pi} \int_{0}^{2 \pi} \mid f\left(\left.x\right|^{p} d x\right\}^{\frac{1}{p}} .\right.
$$

The norm in the space $H_{p}^{(\omega)}$ is defined by

$$
\|f\|_{p}^{(\omega)}:=\|f\|_{p}+A(f, \omega) .
$$

Given the spaces $H_{p}^{(\omega)}$ and $H_{p}^{(v)}$, if $\frac{\omega(t)}{v(t)}$ is nondecreasing , then

$$
H_{p}^{(\omega)} \subseteq H_{p}^{(v)} \subseteq L_{p} \quad p \geq 1
$$

since

$$
\|f\|_{p}^{(v)} \leq \max \left(1, \frac{\omega(2 \pi)}{v(2 \pi)}\right)\|f\|_{p}^{(\omega)} .
$$

Especially, the leading studies related to degree of approximation in $H_{p}^{(\omega)}$ space can be found in [7] and [12].

\section{DEFERRED CESÀro MEAN AND ITS GENERALIZATION}

The concept of deferred Cesàro mean has been given by Agnew as following (see [1]). Let $a=\left(a_{n}\right)$ and $b=\left(b_{n}\right)$ be sequences of nonnegative integers with conditions

$$
a_{n}<b_{n} \quad n=1,2,3, \ldots
$$

and

$$
\lim _{n \rightarrow \infty} b_{n}=+\infty \text {. }
$$

The deferred Cesàro mean, $D_{a}^{b}$, determined by $a$ and $b$

$$
D_{n}=D_{a}^{b}=\frac{S_{a_{n}+1}+S_{a_{n}+2}+\cdots+S_{b_{n}}}{b_{n}-a_{n}}=\frac{1}{b_{n}-a_{n}} \sum_{k=a_{n}+1}^{b_{n}} S_{k},
$$

where $\left(S_{k}\right)$ is a sequence of real or complex numbers. 
Since each $D_{a}^{b}$ with conditions (2.1) and (2.2) satisfies the Silverman-Toeplitz conditions, every $D_{a}^{b}$ is regular. Note that $D_{a}^{b}$ involves, except in case $a_{n}=0$ all $n$, means of deferred elements of $\left(S_{n}\right)$. It is also known that $D_{n-1}^{n}$ is the identity transformation and $D_{0}^{n}$ is the $(C, 1)$ transformation. The basic properties of $D_{a}^{b}$ can be found in [1]. By considering deferred Cesàro means, Değer and Küçükaslan gave the following notations with conditions (2.1) and (2.2) in [9].

Let $\left(p_{n}\right)$ be a positive sequence of real numbers. Then

$$
D_{a}^{b} N_{n}(f ; x)=\frac{1}{P_{0}^{b_{n}-a_{n}-1}} \sum_{m=a_{n}+1}^{b_{n}} p_{b_{n}-m} S_{m}(f ; x),
$$

and

$$
D_{a}^{b} R_{n}(f ; x)=\frac{1}{P_{a_{n}+1}^{b_{n}}} \sum_{m=a_{n}+1}^{b_{n}} p_{m} S_{m}(f ; x)
$$

where

$$
P_{0}^{b_{n}-a_{n}-1}=\sum_{k=0}^{b_{n}-a_{n}-1} p_{k} \neq 0, \quad P_{a_{n}+1}^{b_{n}}=\sum_{k=a_{n}+1}^{b_{n}} p_{k} \neq 0
$$

and

$$
S_{n}(f ; x)=\frac{1}{\pi} \int_{-\pi}^{\pi} f(x+t) D_{n}(t) d t,
$$

in which

$$
D_{n}(t)=\frac{\sin \left(n+\frac{1}{2}\right) t}{2 \sin \left(\frac{t}{2}\right)} .
$$

These two methods are called deferred Woronoi-Nörlund means, $\left(D_{a}^{b} N, p\right)$, and deferred Riesz means, $\left(D_{a}^{b} R, p\right)$, with respect to $S_{m}(f ; x)$, respectively. In case $b_{n}=n$ and $a_{n}=0$, the methods $D_{a}^{b} N_{n}(f ; x)$ and $D_{a}^{b} R_{n}(f ; x)$ give us the classicaly known Woronoi-Nörlund and Riesz means, respectively. Provided that $p_{n}=1$ for all $n(\geq 0)$, both of them yield deferred Cesàro means

$$
D_{a}^{b}(f ; x)=\frac{1}{b_{n}-a_{n}} \sum_{m=a_{n}+1}^{b_{n}} S_{m}(f ; x)
$$

of $S_{m}(f, x)$.

In addition to this, if $b_{n}=n, a_{n}=0$ and $p_{k}=1$ for these two methods, then they coincide with Cesàro method $C_{1}$. In the event that $a_{n}=0,\left(b_{n}\right)$ is a strictly increasing sequence of positive integers with $b(0)=0$ and $p_{k}=1$, then they give us Cesàro submethod which is obtained by deleting a set of rows from Cesàro matrix (see $[3,8,18])$. 
There exist some inclusions that established relation between deferred method and Cesàro method. Before giving this inclusions without detail, we need to give some definition. If $\left(a_{n}\right)$ and $\left(b_{n}\right)$ satisfy, in addition to (2.1) and (2.2), the condition

$$
\frac{a_{n}}{b_{n}-a_{n}}=O(1)
$$

for all $n$, then $\left(D_{a}^{b}\right)$ is properly deferred ; such a transformation is called a proper $\left(D_{a}^{b}\right)$ (see [1]). Therefore we know that

$$
(C, 1) \subset D\left(a_{n}, b_{n}\right) \text { if and only if }\left(D_{a}^{b}\right) \text { is properly deferred, }
$$

and

$$
D\left(a_{n}, n\right) \subset(C, 1) .
$$

On the other hand,

$$
D\left(a_{n}, n\right) \sim(C, 1) \quad \text { if and only if } \quad\left(D_{a}^{b}\right) \quad \text { is properly deferred. }
$$

In 2014, Nayak et al. has studied the rate of convergence problem of Fourier series by deferred Cesàro mean in the generalized Hölder metric $\left(H_{p}^{(w)}\right)$ and gave the following theorem.

Theorem 2 ([17]). Let $v$ and $w$ be moduli of continuity such that $\frac{w}{v}$ is nondecreasing and $f \in H_{p}^{(w)}, p \geq 1$. Let

$$
q_{n}=(2 j+1) p_{n}+2 j
$$

where $j$ is a positive integer. Then

(i) $\left\|D_{n}\left(S_{n}(f ; .)\right)-f(.)\right\|_{p}^{(v)}=O\left(\frac{1}{p_{n}+1}\right)+\frac{O(1)}{\left(p_{n}+1\right)^{2}} \int_{\frac{\pi}{\left(p_{n}+1\right)}}^{\pi} \frac{w(t)}{t^{3} v(t)} d t$.

If in addition $\frac{w(t)}{t v(t)}$ is non-increasing then

$$
\text { (ii })\left\|D_{n}\left(S_{n}(f ; .)\right)-f(.)\right\|_{p}^{(v)}=\frac{O(1)}{\left(p_{n}+1\right)^{2}} \int_{\frac{\pi}{\left(p_{n}+1\right)}}^{\pi} \frac{w(t)}{t^{3} v(t)} d t
$$

and a fortiori

$$
\text { (i i i ) }\left\|D_{n}\left(S_{n}(f ; .)\right)-f(.)\right\|_{p}^{(v)}=O\left(\frac{w\left(\pi /\left(p_{n}+1\right)\right)}{v\left(\pi /\left(p_{n}+1\right)\right)}\right) .
$$

Taking into account of this theorem and the methods given in [9], we shall improve the results in [17] on the degree of approximation by the generalized deferred Cesàro means in the space $H_{p}^{\omega}$. Therefore the results given in [17] are generalized according to the summability method. 


\section{THEOREMS}

In this section we will give two theorems on the degree of approximation by deferred Woronoi-Nörlund means and deferred Riesz means in generalized Hölder metric.

Theorem 3. Let $v$ and $w$ be moduli of continuity such that $\frac{w}{v}$ is nondecreasing and $f \in H_{p}^{(w)}, p \geq 1$. Let $b_{n}=(2 j+1) a_{n}+2 j$ where $j$ is a positive integer. Moreover let $\left(p_{n}\right)$ be a positive sequence and the conditions

$$
\left(b_{n}-a_{n}\right) p_{b_{n}}=O\left(P_{a_{n}+1}^{b_{n}}\right)
$$

and

$$
\sum_{m=a_{n}+1}^{b_{n}-1}\left|\Delta_{m}\left(p_{m}\right)\right|=O\left(\left|p_{b_{n}}-p_{a_{n}+1}\right|\right)
$$

are satisfied where $\Delta_{m}\left(p_{m}\right)=p_{m}-p_{m+1}$. Then

$$
\begin{aligned}
& \left\|D_{a}^{b} R_{n}(f ; .)-f(.)\right\|_{p}^{(v)} \\
& \quad=O\left(1+\frac{\left|p_{b_{n}}-p_{a_{n}+1}\right|}{p_{b_{n}}}\right)\left\{\frac{1}{a_{n}+1}+\frac{1}{\left(a_{n}+1\right)^{2}} \int_{\frac{\pi}{\left(a_{n}+1\right)}}^{\pi} \frac{w(t)}{t^{3} v(t)} d t\right\}
\end{aligned}
$$

Proof. By definition of the deferred Riesz means, we have

$$
D_{a}^{b} R_{n}(f ; .)-f(.)=\frac{1}{P_{a_{n}+1}^{b_{n}}} \sum_{m=a_{n}+1}^{b_{n}} p_{m}\left(S_{m}(f ; .)-f(.)\right) .
$$

By elementary methods, we get

$$
I:=D_{a}^{b} R_{n}(f ; .)-f(.)=\frac{1}{P_{a_{n}+1}^{b_{n}}} \sum_{m=0}^{b_{n}-a_{n}-1} p_{m+a_{n}+1}\left(S_{m+a_{n}+1}(f ; .)-f(.)\right) .
$$

Using Abel's transformation, we see that

$$
\begin{aligned}
I= & \frac{1}{P_{a_{n}+1}^{b_{n}}} \sum_{m=0}^{b_{n}-a_{n}-2}\left[p_{m+a_{n}+1}-p_{m+a_{n}+2}\right]\left[\sum_{k=0}^{m}\left(S_{k+a_{n}+1}(f ; .)-f(.)\right)\right] \\
& +\frac{1}{P_{a_{n}+1}^{b_{n}}} \sum_{k=0}^{b_{n}-a_{n}-1}\left[\left(S_{k+a_{n}+1}(f ; .)-f(.)\right)\right] p_{b_{n}}=: I_{1}+I_{2}
\end{aligned}
$$

By considering the second term in right side of the above equality, we write

$$
I_{2}=\frac{p_{b_{n}}}{P_{a_{n}+1}^{b_{n}}} \frac{b_{n}-a_{n}}{b_{n}-a_{n}} \sum_{k=a_{n}+1}^{b_{n}}\left(S_{k}(f ; .)-f(.)\right)=\frac{p_{b_{n}}\left(b_{n}-a_{n}\right)}{P_{a_{n}+1}^{b_{n}}}\left(D_{a}^{b}\left(S_{n}(f ; .)\right)-f(.)\right) \text {. }
$$


Hence by the condition (3.1) and Theorem 2-(i), we obtain

$$
\begin{aligned}
\left\|I_{2}\right\|_{p}^{(v)} & \leq\left|\frac{p_{b_{n}}\left(b_{n}-a_{n}\right)}{P_{a_{n}+1}^{b_{n}}}\right|\left\|D_{a}^{b}\left(S_{n}(f ; .)\right)-f(.)\right\|_{p}^{(v)} \\
& =O(1)\left\|D_{a}^{b}\left(S_{n}(f ; .)\right)-f(.)\right\|_{p}^{(v)} \\
& =O\left(\frac{1}{a_{n}+1}\right)+\frac{O(1)}{\left(a_{n}+1\right)^{2}} \int_{\frac{\pi}{\left(a_{n}+1\right)}}^{\pi} \frac{w(t)}{t^{3} v(t)} d t .
\end{aligned}
$$

Now let us consider the first term in (3.3). Then we have

$$
\begin{aligned}
I_{1} & =\frac{1}{P_{a_{n}+1}^{b_{n}}} \sum_{m=0}^{b_{n}-a_{n}-2}\left[\sum_{k=0}^{m}\left(S_{k+a_{n}+1}(f ; .)-f(.)\right)\right]\left[p_{m+a_{n}+1}-p_{m+a_{n}+2}\right] \\
& =\frac{1}{P_{a_{n}+1}^{b_{n}}} \sum_{m=a_{n}+1}^{b_{n}-1} \Delta_{m}\left(p_{m}\right) \sum_{k=0}^{m-a_{n}-1}\left(S_{k+a_{n}+1}(f ; .)-f(.)\right) \\
& =\frac{1}{P_{a_{n}+1}^{b_{n}}} \sum_{m=a_{n}+1}^{b_{n}-1} \Delta_{m}\left(p_{m}\right) \frac{m-a_{n}}{m-a_{n}} \sum_{k=a_{n}+1}^{m}\left(S_{k}(f ; .)-f(.)\right) \\
& =\frac{1}{P_{a_{n}+1}^{b_{n}}} \sum_{m=a_{n}+1}^{b_{n}-1}\left(m-a_{n}\right) \Delta_{m}\left(p_{m}\right)\left[D_{a}^{m}\left(S_{n}(f ; .)\right)-f(.)\right]
\end{aligned}
$$

By considering (3.5) and Theorem 2-(i), we write

$$
\begin{aligned}
& \left\|I_{1}\right\|_{p}^{(v)} \leq \frac{1}{P_{a_{n}+1}^{b_{n}}}\left(b_{n}-a_{n}\right) \sum_{m=a_{n}+1}^{b_{n}-1}\left|\Delta_{m}\left(p_{m}\right)\right|\left\|D_{a}^{m}\left(S_{n}(f ; .)\right)-f(.)\right\|_{p}^{(v)} \\
& =\frac{\left(b_{n}-a_{n}\right)}{P_{a_{n}+1}^{b_{n}}}\left\{O\left(\frac{1}{a_{n}+1}\right)+\frac{O(1)}{\left(a_{n}+1\right)^{2}} \int_{\frac{\pi}{\left(a_{n}+1\right)}}^{\pi} \frac{w(t)}{t^{3} v(t)} d t\right\} \sum_{m=a_{n}+1}^{b_{n}-1}\left|\Delta_{m}\left(p_{m}\right)\right| .
\end{aligned}
$$

Owing to condition (3.1) and condition (3.2) in the last term, we obtain

$$
\left\|I_{1}\right\|_{p}^{(v)}=\frac{\left|p_{b_{n}}-p_{a_{n+1}}\right|}{p_{b_{n}}}\left\{O\left(\frac{1}{a_{n}+1}\right)+\frac{O(1)}{\left(a_{n}+1\right)^{2}} \int_{\frac{\pi}{\left(a_{n}+1\right)}}^{\pi} \frac{w(t)}{t^{3} v(t)} d t\right\} .
$$

Combining (3.3), (3.4) and (3.6), we get the desired result . Therefore the proof is completed.

Corollary 1. Under conditions of Theorem 3, if $f \in H_{p}^{(w)}$ for $p \geq 1$ and $\frac{w(t)}{t v(t)}$ is nonincreasing then

(i) $\left\|D_{a}^{b} R_{n}(f ; .)-f(.)\right\|_{p}^{(v)}=O(1)\left(1+\frac{\left|p_{b_{n}}-p_{a_{n}+1}\right|}{p_{b_{n}}}\right) \frac{1}{\left(a_{n}+1\right)^{2}} \int_{\frac{\pi}{\left(a_{n}+1\right)}}^{\pi} \frac{w(t)}{t^{3} v(t)} d t$ 


\section{and furthermore}

(ii) $\left\|D_{a}^{b} R_{n}(f ; .)-f(.)\right\|_{p}^{(v)}=O(1)\left(1+\frac{\left|p_{b_{n}}-p_{a_{n}+1}\right|}{p_{b_{n}}}\right) \frac{w\left(\pi /\left(a_{n}+1\right)\right)}{v\left(\pi /\left(a_{n}+1\right)\right)}$.

Theorem 3 and its corollaries are very important due to the relations between Riesz type deferred method and Riesz method. Let us recall some results establishing these relations given in [9]. Suppose that the sequences $\left(a_{n}\right)$ and $\left(b_{n}\right)$ satisfy the conditions (2.1) and (2.2). If the condition

$$
\frac{p_{1}+p_{2}+\cdots+p_{a_{n}}}{P_{a_{n}+1}^{b_{n}}}=O(1)
$$

satisfy for $\left(p_{n}\right)$, then we shall say that $\left(D_{a}^{b} R, p\right)$ is properly deferred and such a transformation is called a proper $\left(D_{a}^{b} R, p\right)$. We see that if $\left(p_{n}\right)=1$ for all $n$ with the conditions (2.1) and (2.2), then $\left(D_{a}^{b} R, p\right)$ and the condition (3.7) are reduced (D) and the condition (2.3), respectively. In [9], we know that " $(R, p) \subset\left(D_{a}^{b} R, p\right)$ if and only if $\left(D_{a}^{b} R, p\right)$ is proper". On the other hand, we have " $\left(D_{a}^{n} R, p\right) \subset(R, p)$ " and $"\left(D_{a}^{n} R, p\right) \sim(R, p)$ if and only if $\left(D_{a}^{n} R, p\right)$ is proper".

Theorem 4. Let $v$ and $w$ be moduli of continuity such that $\frac{w}{v}$ is nondecreasing and $f \in H_{p}^{(w)}, p \geq 1$. Let

$$
b_{n}=(2 j+1) a_{n}+2 j
$$

where $j$ is a positive integer. Moreover let $\left(p_{n}\right)$ be a positive sequence and the conditions

$$
\left(b_{n}-a_{n}\right) p_{b_{n}-a_{n}-1}=O\left(P_{0}^{b_{n}-a_{n}-1}\right)
$$

and

$$
\sum_{m=a_{n}+1}^{b_{n}-1}\left|\Delta_{m}\left(p_{b_{n}-m}\right)\right|=O\left(\left|p_{b_{n}-a_{n}-1}-p_{0}\right|\right)
$$

are satisfied. Then

$$
\begin{aligned}
& \left\|D_{a}^{b} N_{n}(f ; \cdot)-f(\cdot)\right\|_{p}^{v} \\
& =O\left(\frac{p_{0}+\left|p_{b_{n}-a_{n}-1}-p_{0}\right|}{p_{b_{n}-a_{n}-1}}\right)\left\{\frac{1}{a_{n}+1}+\frac{1}{\left(a_{n}+1\right)^{2}} \int_{\frac{\pi}{\left(a_{n}+1\right)}}^{\pi} \frac{w(t)}{t^{3} v(t)} d t\right\} .
\end{aligned}
$$

Proof. Since

$$
J:=D_{a}^{b} N_{n}(f ; .)-f(.)=\frac{1}{P_{0}^{b_{n}-a_{n}-1}} \sum_{m=0}^{b_{n}-a_{n}-1} p_{b_{n}-m-a_{n}-1}\left(S_{m+a_{n}+1}(f ; .)-f(.)\right),
$$


we get that

$$
\begin{aligned}
J= & \frac{1}{P_{0}^{b_{n}-a_{n}-1}} \sum_{m=0}^{b_{n}-a_{n}-2}\left[\sum_{k=0}^{m}\left(S_{k+a_{n}+1}-f(.)\right)\right]\left[p_{b_{n}-m-a_{n}-1}-p_{b_{n}-m-a_{n}-2}\right] \\
& +\frac{1}{P_{0}^{b_{n}-a_{n}-1}} \sum_{k=0}^{b_{n}-a_{n}-1}\left[\left(S_{k+a_{n}+1}(f ; .)-f(.)\right] p_{0}=: J_{1}+J_{2}\right.
\end{aligned}
$$

by Abel's transformation. Let us consider $J_{2}$. Since

$$
J_{2}=\frac{p_{0}\left(b_{n}-a_{n}\right)}{P_{0}^{b_{n}-a_{n}-1}}\left(D_{a}^{b}\left(S_{n}(f ; .)\right)-f(.)\right),
$$

we have

$$
\left\|J_{2}\right\|_{p}^{(v)} \leq O(1) \frac{p_{0}}{p_{b_{n}-a_{n}-1}}\left\{\frac{1}{a_{n}+1}+\frac{1}{\left(a_{n}+1\right)^{2}} \int_{\frac{\pi}{\left(a_{n}+1\right)}}^{\pi} \frac{w(t)}{t^{3} v(t)} d t\right\}
$$

by considering (3.8) and Theorem 2-(i). Let us estimate $J_{1}$. By elementary methods, we know that

$$
\begin{aligned}
J_{1} & =\frac{1}{P_{0}^{b_{n}-a_{n}-1}} \sum_{m=a_{n}+1}^{b_{n}-1}\left(p_{b_{n}-m}-p_{b_{n}-m-1}\right) \sum_{k=0}^{m-a_{n}-1}\left(S_{k+a_{n}+1}(f ; .)-f(.)\right) \\
& =\frac{1}{P_{0}^{b_{n}-a_{n}-1}} \sum_{m=a_{n}+1}^{b_{n}-1} \Delta_{m}\left(p_{b_{n}-m}\right)\left(m-a_{n}\right)\left[D_{a}^{m}\left(S_{n}(f ; .)\right)-f(.)\right] .
\end{aligned}
$$

Taking into account of (3.8), (3.9) and Theorem 2-(i), we write

$$
\left\|J_{1}\right\|_{p}^{(v)} \leq O\left(\frac{\left|p_{b_{n}-a_{n}-1}-p_{0}\right|}{p_{b_{n}-a_{n}-1}}\right)\left\{\frac{1}{a_{n}+1}+\frac{1}{\left(a_{n}+1\right)^{2}} \int_{\frac{\pi}{\left(a_{n}+1\right)}}^{\pi} \frac{w(t)}{t^{3} v(t)} d t\right\} .
$$

Therefore, we get (3.10) by collecting of (3.11)-(3.13).

Corollary 2. Under conditions of Theorem 4, if $f \in H_{p}^{(w)}, p \geq 1$ and $\frac{w(t)}{t v(t)}$ is nonincreasing then

(i) $\left\|D_{a}^{b} N_{n}(f ; .)-f(.)\right\|_{p}^{(v)}=O(1) \frac{1}{\left(a_{n}+1\right)^{2}}\left(\frac{p_{0}+\left|p_{b_{n}-a_{n}-1}-p_{0}\right|}{p_{b_{n}-a_{n}-1}}\right) \int_{\frac{\pi}{\left(a_{n}+1\right)}}^{\pi} \frac{w(t)}{t^{3} v(t)} d t$ and furthermore

(ii) $\left\|D_{a}^{b} N_{n}(f ; .)-f(.)\right\|_{p}^{(v)}=O(1)\left(\frac{w\left(\pi /\left(a_{n}+1\right)\right)}{v\left(\pi /\left(a_{n}+1\right)\right)}\right)\left(\frac{p_{0}+\left|p_{b_{n}-a_{n}-1}-p_{0}\right|}{p_{b_{n}-a_{n}-1}}\right)$.

Remark 1 . If we take $p_{k}=1$ in Theorem 3, Theorem 4, Corollary 1 and Corollary 2 , then all of results coincide with Theorem 2 . 


\section{CONClusion}

Several studies have been carried out on the degree of approach in the $H_{p}^{(w)}$ spaces. The means used in these studies include the results obtained by approaching to functions in this space using the classically known Cesàro, Woronoi-Nörlund and Riesz means. These results depend either directly related to the degree of polynomials, or to the upper and lower limits of the method such as deferred Cesàro means. In this study we have shown that how the speed of the approach is being affected, based on the sequences that determines the Woronoi-Nörlund and Riesz means by considering the deferred type of the Woronoi-Nörlund and Riesz mean introduced in [9]. It is evident that the results generalized previous work on these spaces.

\section{REFERENCES}

[1] R. P. Agnew, "On deferred Cesáro means," Ann. Math. (2), vol. 33, no. 3, pp. 413-421, 1932, doi: $10.2307 / 1968524$.

[2] G. Alexits, "Convergence problems of orthogonal series," (International Series of Monographs on Pure and Applied Mathematics. Vol. 20.) New York-Oxford-London-Paris: Pergamon Press. IX, 350 p. (1961), 1961.

[3] D. H. Armitage and I. J. Maddox, "A new type of Cesàro mean," Analysis, vol. 9, no. 1-2, pp. 195-204, 1989.

[4] P. Chandra, "On the generalized Fejer means in the metric of Hölder space," Math. Nachr, vol. 109, pp. 39-45, 1982, doi: 10.1002/mana.19821090105.

[5] P. Chandra, "Trigonometric approximation of functions in $L_{p}$-norm," J. Math. Anal. Appl, vol. 275, no. 1, pp. 13-26, 2002, doi: 10.1016/S0022-247X(02)00211-1.

[6] G. Das, T. Ghosh, and B. Ray, "Degree of approximation of functions by their Fourier series in the generalized Hölder metric," Proc. Indian Acad. Sci., Math. Sci, vol. 106, no. 2, pp. 139-153, 1996, doi: 10.1007/BF02837167.

[7] G. Das, A. Nath, and B. K. Ray, "An estimate of the rate of convergence of Fourier series in the generalized Hölder metric," in Analysis and applications. Outcome of the conference dedicated to Professor Tribikram Pati on the occasion of his 70th birthday, Ujjain, India, 1999. New Delhi: Narosa Publishing House; Boca Raton, FL: Chapman and Hall/CRC, 2002, pp. 43-60.

[8] U. Değer and M. Kaya, "On the approximation by Cesáro submethod," Palest. J. Math, vol. 4, no. 1, pp. 44-56, 2015.

[9] U. Değer and M. Küçükaslan, "A generalization of deferred Cesàro means and some of their applications," J. Inequal. Appl, vol. 2015, p. 16, 2015, doi: 10.1186/s13660-014-0532-0.

[10] K. Khatri and V. N. Mishra, "Degree of approximation by the $\left(T \cdot E^{1}\right)$ means of Conjugate Series of Fourier Series in the Hölder metric," Iran J Sci Technol Trans Sci, vol. 2017, p. 9, 2017, doi: https://doi.org/10.1007/s40995-017-0272-3.

[11] L. Leindler, "Generalizations of Proessdorf's theorems," Stud. Sci. Math. Hung, vol. 14, pp. 431439, 1979

[12] L. Leindler, "A relaxed estimate of the degree of approximation by Fourier series in generalized Hölder metric," Anal. Math, vol. 35, no. 1, pp. 51-60, 2009, doi: 10.1007/s10476-009-0106-6.

[13] L. Leindler, "Trigonometric approximation in $L_{p}$-norm," J. Math. Anal. Appl, vol. 302, no. 1, pp. 129-136, 2005, doi: 10.1016/j.jmaa.2004.07.049.

[14] S. Mazhar and V. Totik, "Approximation of continuous functions by $T$-means of Fourier series," J. Approx. Theory, vol. 60, no. 2, pp. 174-182, 1990, doi: 10.1016/0021-9045(90)90082-2. 
[15] V. N. Mishra and L. N. Mishra, "Trigonometric approximation of signals (functions) in $L_{p}$-norm," Int. J. Contemp. Math. Sci, vol. 7, no. 17-20, pp. 909-918, 2012.

[16] R. Mohapatra and P. Chandra, "Degree of approximation of functions in the Hölder metric," Acta Math. Hung, vol. 41, pp. 67-76, 1983, doi: 10.1007/BF01994063.

[17] L. Nayak, G. Das, and B. Ray, "An estimate of the rate of convergence of Fourier series in the generalized Hölder metric by deferred Cesàro mean," J. Math. Anal. Appl, vol. 420, no. 1, pp. 563-575, 2014, doi: 10.1016/j.jmaa.2014.06.001.

[18] J. A. Osikiewicz, "Equivalence results for Cesàro submethods," Analysis, München, vol. 20, no. 1, pp. 35-43, 2000, doi: 10.1524/anly.2000.20.1.35.

[19] S. Prössdorf, "Zur konvergenz der fourierreihen hölderstetiger funktionen," Mathematische Nachrichten, vol. 69, no. 1, pp. 7-14, 1975, doi: 10.1002/mana.19750690102. [Online]. Available: http://dx.doi.org/10.1002/mana.19750690102

[20] E. Quade, "Trigonometric approximation in the mean," Duke Math. J, vol. 3, pp. 529-543, 1937, doi: 10.1215/S0012-7094-37-00342-9.

Authors' addresses

Uğur Değer

Mersin University, Faculty of Science and Literature, Department of Mathematics, 33343, Mersin, Turkey

E-mail address: degpar@hotmail.com(udeger@mersin.edu.tr)

\section{Hilal Bayindir}

Mersin University, Institute of Science and Literature, Department of Mathematics, 33343, Mersin, Turkey

E-mail address: hilalbayindir2@gmail.com 